\title{
Electroresponsive and cell-affinitive polydopamine/ polypyrrole composite microcapsules with a dual-function of on-demand drug delivery and cell stimulation for electrical therapy
}

\author{
Chaoming Xie ${ }^{1}$, Pengfei $\mathrm{Li}^{1}, \mathrm{Lu} \mathrm{Han}^{1}$, Zhenming Wang ${ }^{1}$, Ting Zhou ${ }^{1}$, Weili Deng ${ }^{1}$, Kefeng Wang ${ }^{2}$ \\ and Xiong $\mathrm{Lu}^{1,2}$
}

Electrical therapy has attracted significant attention because it can modulate cell behaviors and accelerate tissue repair. However, the effectiveness of electrical therapy is limited. This work develops electroresponsive and conductive polydopaminepolypyrrole microcapsules (PDA-PPy-MCs) on titanium surfaces using electrochemical deposition. During the electrochemical process, PDA and dexamethasone (DEX) as the anion are doped into a PPy backbone to neutralize its positive charge. PDA-PPy-MCs possess the cell affinity of PDA, the microstructure of MCs and the electroresponsive capability of PPy. The incorporation of PDA promotes the conductivity and adhesive strength of PPy. PDA-PPy-MCs can respond to electrical signals to release DEX on demand because of the redox behavior of PPy. The microstructure and PDA improve the drug-loading capability of PDA-PPy-MCs. A high-throughput bone marrow stromal cell (BMSC) culture system is designed to study the synergistic effects of composition, microstructure and electrical stimulation on cell behavior. The results indicate that PDA and the microporous structure not only enhance the biocompatibility of PDA-PPy-MC but also strengthen the effect of electrical stimulation. In vivo implantation shows that PDA-PPy-MCs have good biocompatibility. The high cell affinity, microstructure, conductivity and ability to control drug delivery using electrical signals make PDA-PPy-MCs a promising candidate for an on-demand drug delivery and electrical therapy system.

NPG Asia Materials (2017) 9, e358; doi:10.1038/am.2017.16; published online 10 March 2017

\section{INTRODUCTION}

Electrical therapy has in recent years been recognized as an alternative medical treatment for tissue repair and has been used to treat various diseases, such as Parkinson's disease, diabetes, obesity and hypertension. ${ }^{1}$ Electrical therapy modulates cell behaviors, including adhesion, migration, proliferation and differentiation, and regulates tissue functions by applying electrical signals. ${ }^{2}$ Electrical therapy can reduce side effects and immunogenic responses in host tissue. However, conventional electrical therapy is a chronic biophysical treatment and its effectiveness is limited. ${ }^{3}$ Thus, it is imperative to improve the precision and effectiveness of electrical therapy. On-demand drug-delivery systems, which deliver drugs according to patient needs, have attracted significant attention as they can improve tissue regeneration and reduce the risk of complications. ${ }^{4}$ Smart materials are the primary components of on-demand drug-delivery systems, which respond to various stimulations, such as near-infrared, UV light, magnetic, ultrasonic and electrical stimulation (ES), and consequently trigger drug release.
Among these materials, electroresponsive materials have unique advantages that enable precise control of drug delivery using a durative or pulsatile potential applied on electrical therapy devices with complex geometries. ${ }^{5}$

Polypyrrole (PPy) is a widely used electroresponsive material that has the capability to load and release drugs using an electrical switch between the oxidative and reductive state. ${ }^{6}$ Although PPy for drug delivery has been well studied, challenges remain for the practical application of PPy because PPy has two shortcomings. First, the drug-loading efficiency of PPy is limited. ${ }^{7}$ There are two methods to improve the drug-loading efficiency of PPy. One is doping a component with a high surface area, such as carbon nanotubes (CNTs) and graphene oxide (GO), to facilitate drug absorption into the doped PPy. ${ }^{8}$ The drugs are loaded on the surfaces of the CNTs and GO by physical attraction. ${ }^{9}$ Another method is to directly produce a micro/nanoporous PPy with high surface area films on electrical therapy devices. ${ }^{10}$ The general procedure to obtain a microporous PPy involves depositing PPy films on sacrificial templates, such as

\footnotetext{
${ }^{1}$ Key Laboratory of Advanced Technologies of Materials, Ministry of Education, School of Materials Science and Engineering, Southwest Jiaotong University, Sichuan, China and ${ }^{2}$ National Engineering Research Center for Biomaterials, Genome Research Center for Biomaterials, Sichuan University, Sichuan, China

Correspondence: Professor X Lu, Key Laboratory of Advanced Technologies of Materials, Ministry of Education, School of Materials Science and Engineering, Southwest Jiaotong University, Chengdu, Sichuan 610031, China.

E-mail: luxiong_2004@163.com

Received 27 August 2016; revised 20 December 2016; accepted 27 December 2016
} 
polystyrene microspheres (PS-MS) ${ }^{11}$ and poly(methyl methacrylate) colloids, ${ }^{12}$ and then removing the templates.

Second, PPy is lacking in cell affinity, although PPy is already widely used in electrodes for cell stimulation. Many researchers have also reported that PPy has insufficient biocompatibility for cell adhesion. Aznar-Cervantes et al. ${ }^{13}$ coated PPy on the surfaces of silk fibroin scaffolds and found that fewer mesenchymal stem cells adhered to the PPy-coated scaffolds. Ateh et al. ${ }^{14}$ reported that PPy substrates have poor capability to support bovine aortic endothelial cell adhesion. Researchers have attempted to incorporate natural biomolecules into PPy to improve the biocompatibility of the PPy. Meng et al. ${ }^{15}$ prepared a conductive PPy/heparin composite substrate to promote osteoblast adhesion and growth through ES. Shi et al. ${ }^{16}$ developed conductive, nanoporous cellulose hydrogels with PPy nanoparticles, which could induce cellular responses by electric stimulation for nerve regeneration.

Incorporating polydopamine (PDA) biomolecules into $\mathrm{PPy}$ is a possible solution for improving the cell affinity of PPy. PDA has a similar molecular structure to that of mussel adhesive proteins ${ }^{17}$ and has attracted great interest as a biomolecule for the surface modification of biomedical implants to improve cell adherence. ${ }^{18}$ PDA microcapsules (PDA-MCs) were recently developed as a promising candidate for drug delivery as they have an adjustable surface area to enable a high drug-loading ratio, have abundant functional groups to bind drugs and are non-cytotoxic. ${ }^{19,20}$ PDA-MCs are generally prepared by coating PDA on sacrificial templates, such as silica $\left(\mathrm{SiO}_{2}\right)$ particles, PS-MS and calcium carbonate particles. $^{21,22}$ Cui et al. ${ }^{19}$ prepared PDA-MCs using a dimethyldiethoxysilane emulsion template and used PDA-MCs for loading doxorubicin. Wang et al. ${ }^{23}$ used PS-MS as a template to fabricate PDA-MC layer-by-layer films and then immobilized bone morphogenetic protein-2 on the PDA-MC surfaces. However, PDA-MC is not conductive, which limits its applications in electrical therapy.

In this study, electroresponsive and conductive PDA-PPy-MCs are synthesized for controllable drug delivery and ES of cells using electrochemical deposition (ED) with the aid of a sulfonated PS-MS (SPS-MS) sacrificial template. ED is a facile method to synthesize PDA and PPy on electrical therapy devices with complex geometry that avoids the uncontrollable self-polymerization of PDA and PPy in solution. Dexamethasone (DEX) was selected as a model drug to be doped into the MCs during the ED process. Compared with previously reported PDA or PPy composite, the current PDA-PPy-MCs have several advantages. First, PDA doping improves the cell affinity of PDA-PPy-MCs. Together with its porous structure, the PDA-PPy-MCs favors cell attachment and growth. Second, due to the drug affinity of PDA and porous structures of MCs, the electroresponsive PDA-PPyMCs have a high drug-loading capability and can deliver drugs on demand (in response to ES signals) due to the redox property of PPy. Third, PDA doping promotes the conductivity of PDA-PPy-MCs. The conductivity, adhesion strength, drug-loading ratio and on-demand drug release profiles were evaluated. The behavior of bone marrow stromal cell (BMSC) on the PDA-PPy-MCs under high-throughput ES conditions was investigated in vitro. The biocompatibility of the PDAPPy-MCs was also evaluated in vivo.

\section{EXPERIMENTAL PROCEDURES}

\section{Preparation of sulfonated polystyrene microspheres (SPS-MS)}

SPS-MS were prepared using a dispersion polymerization method reported in our previous work. ${ }^{23}$ First, azodiisobutyronitrile $(0.3 \mathrm{~g}$; Sigma, St Louis, MO, USA), polyvinyl pyrrolidone (3 g; Sigma), styrene (30 g; Sigma), and ethanol (200 g; Sigma) were added into a flask with stirring under a nitrogen atmosphere at $65{ }^{\circ} \mathrm{C}$ and reacted for $12 \mathrm{~h}$. Second, the suspension was collected and centrifuged at 8000 r.p.m. for $10 \mathrm{~min}$, and the PS-MS were obtained by washing with deionized (DI) water five times. Afterward, the SPS-MS were prepared by dispersing PS-MS into a $98 \%$ sulfuric acid solution $(100 \mathrm{ml})$ with stirring at $40{ }^{\circ} \mathrm{C}$ for $24 \mathrm{~h}$. Finally, the SPS-MS were obtained by centrifuging (8000 r.p.m.) and washing with ethanol and DI water three times.

\section{Preparation of PDA-PPy-MCs}

SPS-MS assembled on the surface of Ti foil $(1 \times 1 \mathrm{~cm})$ and three-dimensional (3D) printed porous $\mathrm{Ti}(1 \times 1 \times 1 \mathrm{~cm}$, Sailing Science and Technology, Chongqing, China) (SPS-MS-Ti) was prepared by immersing the Ti into an SPS-MS solution $\left(5 \mathrm{mg} \mathrm{ml}^{-1}\right)$ for $24 \mathrm{~h}$, followed by drying at $60^{\circ} \mathrm{C}$.

PDA-PPy-MCs were prepared using ED in a three-electrode cell, followed by tetrahydrofuran (THF) etching. During the ED process, the working, counter and reference electrodes were the SPS-MS-Ti, Pt plate and saturated calomel electrode, respectively. The electrolyte was an aqueous solution containing pyrrole (Py, $2 \mathrm{~mm}$; Sigma), dopamine (DA; $2 \mathrm{mg} \mathrm{ml}^{-1}$; Sigma) and DEX ( $2 \mathrm{mg} \mathrm{ml}^{-1}$; Meilune, Dalian, China). The DA/Py molar ratio was 0.52 . The ED was carried out by cycling the potential between 0 and $0.8 \mathrm{~V}$ vs the saturated calomel electrode at a scan rate of $50 \mathrm{mV} \mathrm{s}^{-1}$ for 300 cycles (total time was $4800 \mathrm{~s}$ ). After ED, the SPS-MS template was removed by immersing the samples in THF for $24 \mathrm{~h}$. During the preparation process, three different ED durations (1600, 3200 and 4800 s) and six DA/Py molar ratios $(0,0.13,0.26,0.39,0.52$ and 0.65$)$ were used to prepare the PDA-PPy-MCs with different polymerization durations and DA/Py molar ratios, respectively. PDA-PPy films and PPy films were prepared without the SPS-MS template.

\section{Characterization of PDA-PPy-MCs}

A scanning electron microscope (SEM; JSM 6390, JEOL, Tokyo, Japan) and transmission electron microscope (TEM; JSM 2100F, JEOL, Tokyo, Japan) were used to observe the morphology of the PDA-PPy-MCs. Fourier transform-infrared spectroscopy (FT-IR; Nicolet 5700, Thermo, Waltham, MA, USA) was used to determine the chemical composition of the PDA-PPy-MCs. A universal testing machine (Instron 5567, Norwood, MA, USA) was used to measure the adhesive strengths of the PDA-PPy films with different DA/Py molar ratios. A four-probe resistivity test system (SZ-82, Jingge Electronics, Suzhou, China) was used to measure the conductivity of the PDA-PPy films with different DA/Py molar ratios.

\section{Electrically stimulated drug delivery}

The electrically stimulated DEX release from the samples with different surface structures (PPy films, PDA-PPy films and PDA-PPy-MCs), different DA/Py molar ratios and different polymerization durations were tested in a two-electrode cell. The working electrode was a DEX-containing Ti foil, and the counter electrode was a Pt plate. The electrolyte $(4 \mathrm{ml})$ was a phosphate-buffered solution $(0.01 \mathrm{M})$. The ES was carried out using a constant cathodic potential of $-1 \mathrm{~V}$ for $120 \mathrm{~min}$. To investigate the effect of the ES potential on the amount of DEX released, three different ES potentials $(-0.5,-1.0$ and $-1.5 \mathrm{~V})$ were applied for a 10 -min stimulation. To characterize the on-demand DEX delivery profile from the PDA-PPy-MCs, pulse potentials were used to stimulate the samples for $70 \mathrm{~min}$ (in each cycle, $0 \mathrm{~V}$ was applied for $10 \mathrm{~min}$ and $-1 \mathrm{~V}$ was applied for $10 \mathrm{~min}$ ). The electrolyte was collected and replaced with fresh phosphate-buffered solution every $10 \mathrm{~min}$. The amount of DEX released was determined by measuring the absorbance of the collected phosphate-buffered solution at $242 \mathrm{~nm}$ using a UV-Vis spectrophotometer (Lambda 35, PerkinElmer, Hopkinton, MA, USA). The statistical analysis is detailed in the Supplementary Information.

\section{High-throughput stimulation of BMSCs}

BMSCs were cultured on the surfaces of pure Ti, PPy films, PDA-PPy films and PDA-PPy-MCs under different ES potentials (0, 300, 600 and $900 \mathrm{mV})$ using a homemade high-throughput ES device (Supplementary Information and Supplementary Figure S1). The ES was applied for $30 \mathrm{~min}$ every day after BMSCs had adhered on the surfaces on the first day. The morphology and proliferation of the BMSCs in the different groups were evaluated 


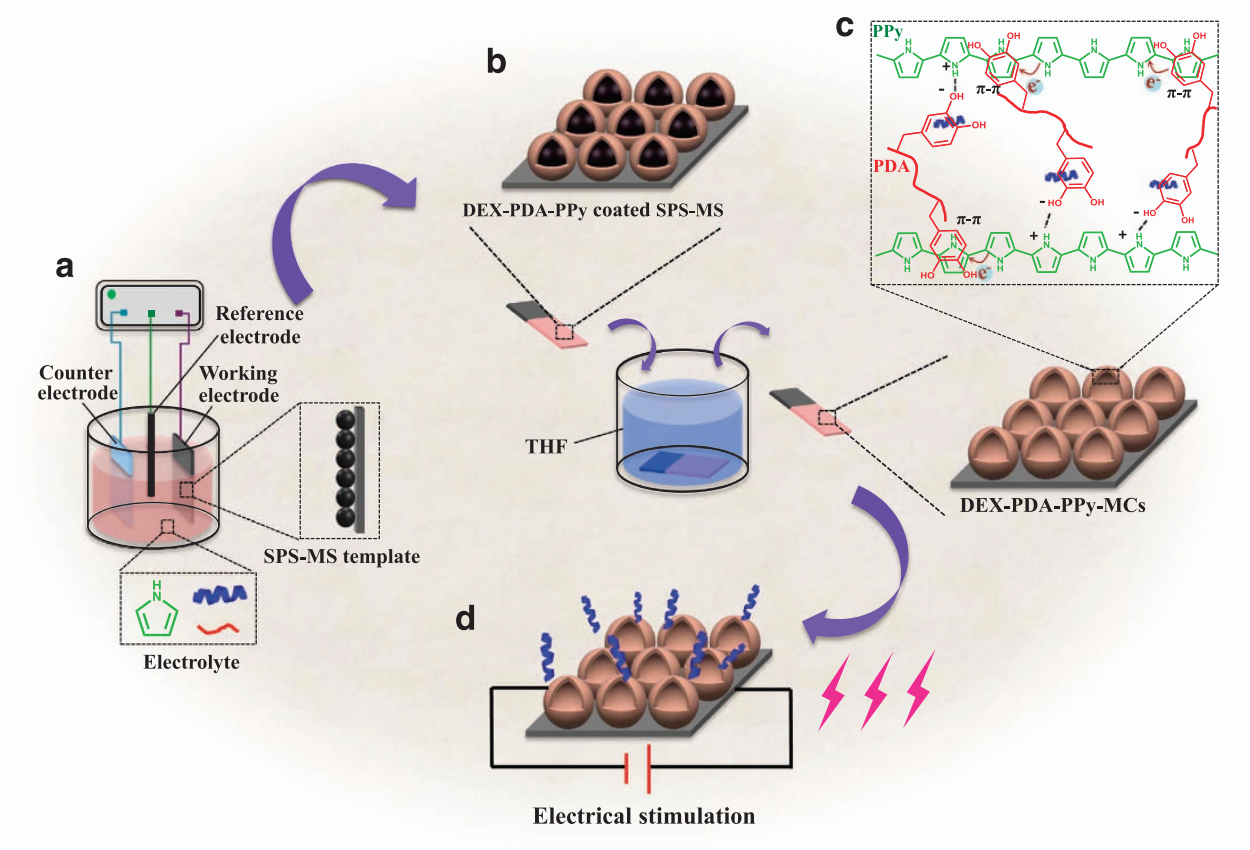

Pyrrole (Py) Dopamine (DA) N D Dexamethasone (DEX)

Sulfonated polystyrene microspheres (SPS-MS)

Scheme 1 Schematic representation of the preparation process of DEX-loaded PDA-PPy-MCs. (a) Electrochemical deposition process. (b) Removing the SPS-MS template by THF etching, (c) PDA and PPy interact each other through hydrogen bonding and $\pi-\pi$ interaction. (d) Drug release by ES.

using fluorescence staining and a Cell Counting Kit-8 (CCK-8; Dojindo, Tokyo, Japan) assay after 3 and 7 days in the culture, respectively. The differentiation of the BMSCs into different groups was evaluated using an alkaline phosphatase (ALP) activity assay (Jiancheng Bioengineering Institute, Nanjing, China) after 14 days in culture. Detailed experimental processes are provided in the Supplementary Information.

\section{Animal experiments}

PPy films, PDA PPy films and PDA-PPy-MCs were prepared on the surfaces of porous $\mathrm{Ti}(\Phi 10 \times 3 \mathrm{~mm})$. Subsequently, the porous Ti, PPy films, PDA-PPy films and PDA-PPy-MC-coated porous Ti were subcutaneously implanted into four 8-week-old Sprague-Dawley rats to evaluate their in vivo biocompatibility. The surgery was performed as previously reported. ${ }^{24}$ After 4 weeks of implantation, all samples were harvested for methylene blue and basic fuchsin staining and then observed using a light microscope (BX63, Olympus, Tokyo, Japan). Detailed experimental processes are provided in the Supplementary Information.

\section{RESULTS}

\section{Synthesis of PDA-PPy-MCs}

The PDA composite MCs were synthesized in three steps. (1) SPS-MS were assembled on the surfaces of Ti (SPS-MS-Ti) to form a sacrificial template layer. (2) PDA has a negative charge due to its catechol groups, ${ }^{25}$ and therefore PDA works as the anion to dope into the backbone of PPy. PDA-PPy composite coatings were polymerized on the surfaces of the SPS-MS-Ti through electrochemical oxidative polymerization (Scheme 1a). 3) PDA-PPy-MCs were obtained after removing the SPS-MS template by THF etching. DEX-loaded PDA-PPy-MCs were prepared using the same methods and using a DA-DEX complex during the ED process. First, DA and DEX were mixed to form a DA-DEX complex. Second, DA-DEX was co-doped into the PPy backbone to neutralize its positive charge with DEX undergoing electrochemical oxidation (Scheme 1b). ${ }^{26}$ PDA and PPy interact with each other through hydrogen bonding and a $\pi-\pi$ interaction (Scheme 1c). ${ }^{27}$ Finally, when ES is applied, the PDA-PPy-MCs are under an electrochemical reductive condition and DEX is released as the charge is excluded from the neutralized PPy backbone (Scheme 1d). The drug doping and release behavior of PDA-PPy-MCs are dependent on the redox property of PPy. The backbone of PPy lacks electrons under oxidative conditions in the polymerization process, which needs anions to balance its positive charge (Supplementary Information and Supplementary Figure S2). ${ }^{28}$ Thus, negatively charged drugs are able to dope into PPy under oxidative conditions. When under reductive conditions, the backbone of PPy is neutralized; therefore, the anions are pushed out from the PPy backbone.

\section{Morphology of PDA-PPy-MCs}

The $3 \mathrm{D}$ printed porous $\mathrm{Ti}$ schematic representation is shown in Figure 1a. The pores of the $3 \mathrm{D}$ printed porous $\mathrm{Ti}$ have a diameter of $\sim 1 \mathrm{~mm}$ (Figure $1 \mathrm{~b}$ ). The SPS-MS covered the surfaces of the 3D printed porous Ti (Figure 1c). A magnified micrograph showed that the SPS-MS were well ordered and that their size was $\sim 2 \mu \mathrm{m}$ (Figure 1d). The surface of the SPS-MS was rough because of the sulfonated modification, which facilitates the polymerization of PDA-PPy on the surface of the SPS-MS. ${ }^{23}$ After the ED, there are many nanoparticles (NPs) on the surfaces of SPS-MS because PDA and PPy were first polymerized to NPs and further aggregated to form coatings during the polymerization process (Figures 1e and f). ${ }^{29,30}$ PDA-PPy-MCs exhibited a porous structure after the removal of the SPS-MPs template (Figures $1 \mathrm{~g}$ and $\mathrm{h}$ ), which is beneficial for a high drug-loading ratio and cell adhesion. Moreover, the morphologies of the PDA-PPy-MCs are not significantly different before and after DEX-loading (Supplementary Information and Supplementary Figure S3). This is because DEX is a small molecule that does not affect the morphologies of PDA-PPy-MCs after loading. 

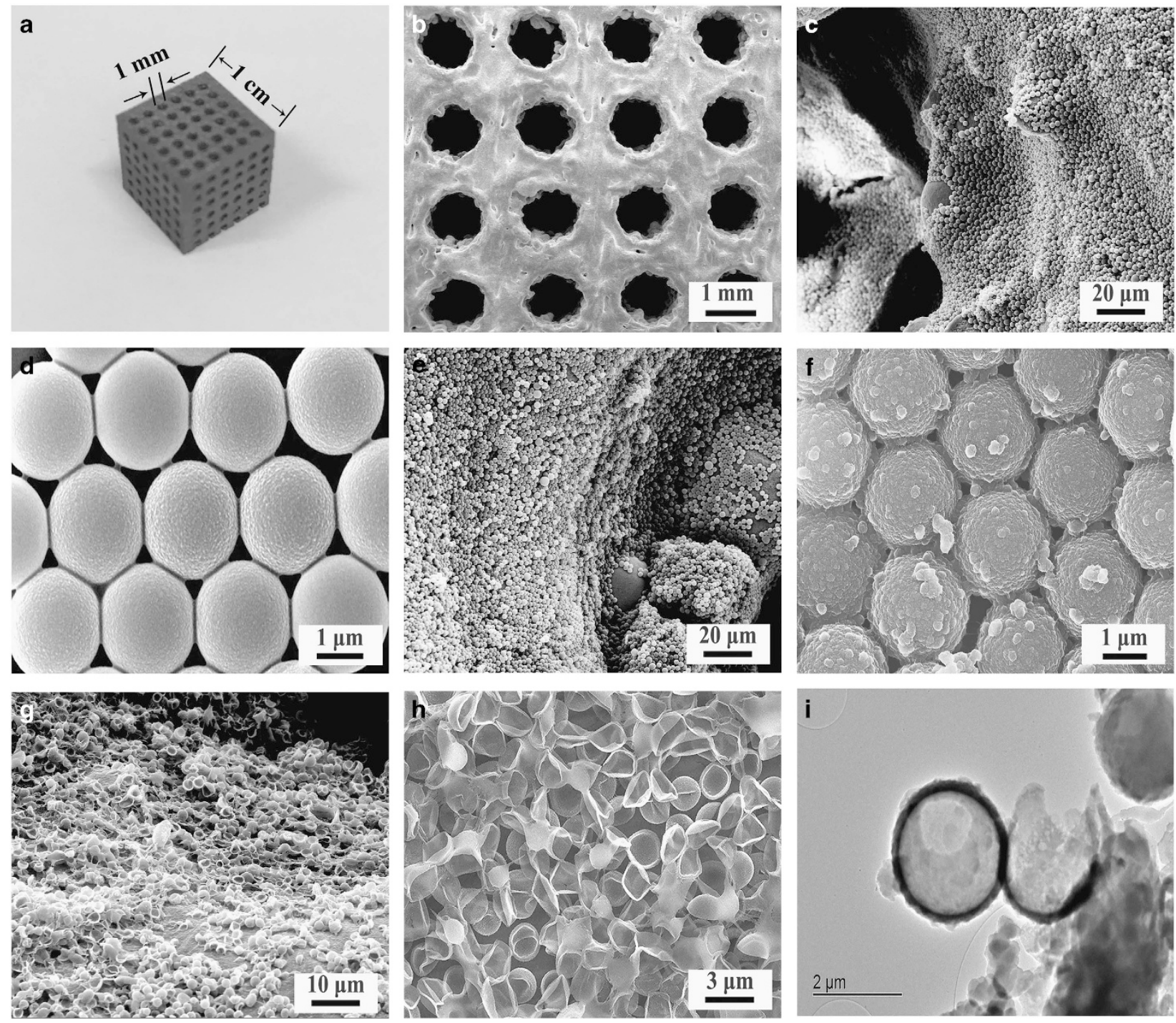

Figure 1 (a) The digital image of 3D printed porous Ti. SEM micrographs of (b) 3D printed porous Ti; (c) SPS-MS template on the surface of the porous Ti, (d) magnified micrograph of c; (e) SPS-MS with PDA-PPy coatings, (f) magnified micrograph of e; (g) PDA-PPy-MCs after removal of the template, (h) magnified micrograph of (g). (i) TEM image of PDA-PPy-MCs.

TEM imaging showed that the thickness of the MC was approximately $200 \mathrm{~nm}$ (Figure 1i). Compared with the FT-IR spectrum of the PPy-MCs, there are two additional peaks in the FT-IR spectrum of the PPy-PDA-MCs. The two peaks at 1518 and $1299 \mathrm{~cm}^{-1}$ are associated with the $\mathrm{N}-\mathrm{H}$ shearing vibration and $\mathrm{C}-\mathrm{O}$ stretching vibration, respectively, indicating the presence of PDA in the PDA-PPy-MCs (Supplementary Information and Supplementary Figure S4).

\section{Conductivity of PDA-PPy-films}

Incorporation of PDA into PDA-PPy-films improves their conductivity compared with the pure PPy-MC (Figure 2). The conductivity of pure PPy was only $3.2 \times 10^{-3} \mathrm{~S} \mathrm{~cm}^{-1}$. The conductivity jumped to $5.8 \times 10^{-2} \mathrm{~S} \mathrm{~cm}^{-1}$ when the DA/Py molar ratio was 0.13 . However, the conductivity decreased to $5.9 \times 10^{-3} \mathrm{~S} \mathrm{~cm}^{-1}$ when further increasing the DA/Py molar ratio from 0.13 to 0.65 , indicating that the higher DA/Py molar ratio is not beneficial for improvement of the conductivity of PDA-PPy-films.

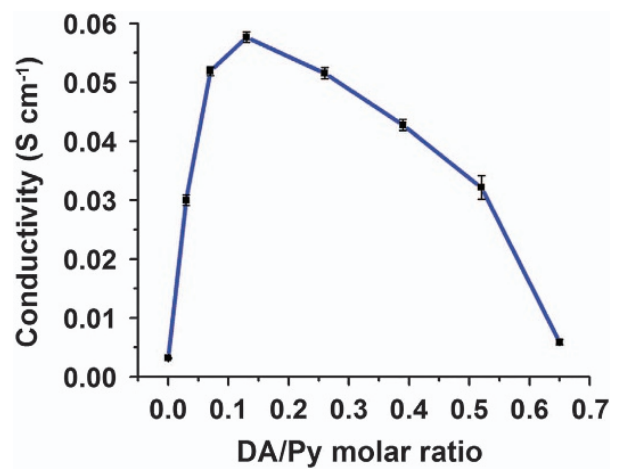

Figure 2 Conductivity of PDA-PPy-films with different DA/Py molar ratios.

Adhesion strength of PDA-PPy-films

An increase in PDA content improves the adhesion strength of the PDA-PPy-films (Figure 3). The adhesion strength of the PDA-PPy 


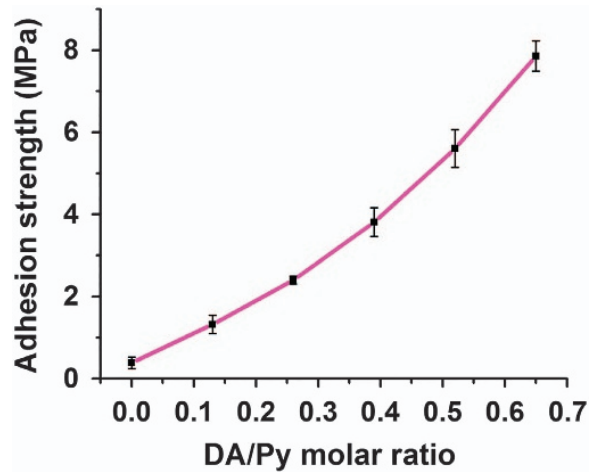

Figure 3 Adhesion strength of PDA-PPy-films with different DA/Py molar ratios.

films increased from 0.38 to $7.86 \mathrm{MPa}$ when the DA/Py molar ratio was increased from 0 to 0.65 . This is because PDA contains many functional groups, such as catechol and amine groups that can react with a variety of substrates to enhance the adhesion strength between the coatings and substrate surface. ${ }^{27}$ Moreover, the thickness of the PDA-PPy-films also increased from 2 to $12 \mu \mathrm{m}$ with the increase in the DA/Py molar ratio (Supplementary Information and Supplementary Figure S5). The adhesion strength of PDA-PPy-MCs was shown in Supplementary Information (Supplementary Figure S6).

\section{Drug-loading capability of PDA-PPy-MCs}

A microporous structure is beneficial to enhance the available drug-loading capability of MCs. The available drug-loading capability was characterized by the cumulative release amount (CRA) of DEX from the MCs. The three DEX release profiles almost reached a plateau after $100 \mathrm{~min}$ of ES, which indicated that the DEX achieved the maximum CRA (Figure 4a). The maximum CRA of DEX from the PPy films, PDA-PPy-films and PDA-PPy-MCs was 15.8 \pm 2.3 , $65.4 \pm 4.2$ and $162.9 \pm 5.7 \mu \mathrm{g} \mathrm{cm}^{-2}$, respectively. The CRA of DEX from the PDA-PPy-MCs was significantly higher than that from the dense PDA-PPy-films and PPy-films, suggesting that the microporous structure of the MCs improved their available drug-loading capability.

Increasing the DA content further enhances the available drug-loading capability of the PDA-PPy-MCs (Figure 4b). The maximum CRA of DEX from PDA-PPy-MCs with the DA/Py molar ratio of $0,0.13,0.26,0.39,0.52$ and 0.65 was $46.0 \pm 0.8,127.1 \pm 1.1$, $143.1 \pm 6.7,158.8 \pm 0.9,162.9 \pm 5.7$ and $154.2 \pm 1.4 \mu \mathrm{g} \mathrm{cm}^{-2}$, respectively. The CRA of DEX from PPy-MCs with PDA was significant higher than that of those without PDA (0 DA/Py molar ratio). However, when the DA/Py molar ratio increased from 0.39 to 0.65 , the DEX CRA showed no significant change.

Increasing polymerization time also increases the available drug-loading capability of PDA-PPy-MCs. As shown in Figure 4c, the CRA of DEX increased nearly threefold when the polymerization time increased from 1600 to $4800 \mathrm{~s}$. The maximum CRA of DEX from PDA-PPy-MCs with 1600,3200 and $4800 \mathrm{~s}$ of polymerization time was $73.7 \pm 7.2,98.9 \pm 6.7$ and $162.9 \pm 5.7 \mu \mathrm{g} \mathrm{cm}^{-2}$, respectively. This is because the increase in polymerization time results in an increase in the thickness of the MCs, and consequently more drugs can be loaded. The TEM images showed that the thickness of PDA-PPy-MCs increased from 50 to $200 \mathrm{~nm}$ when the polymerization time increased from 1600 to $4800 \mathrm{~s}$ (Supplementary Information and Supplementary Figure S7).
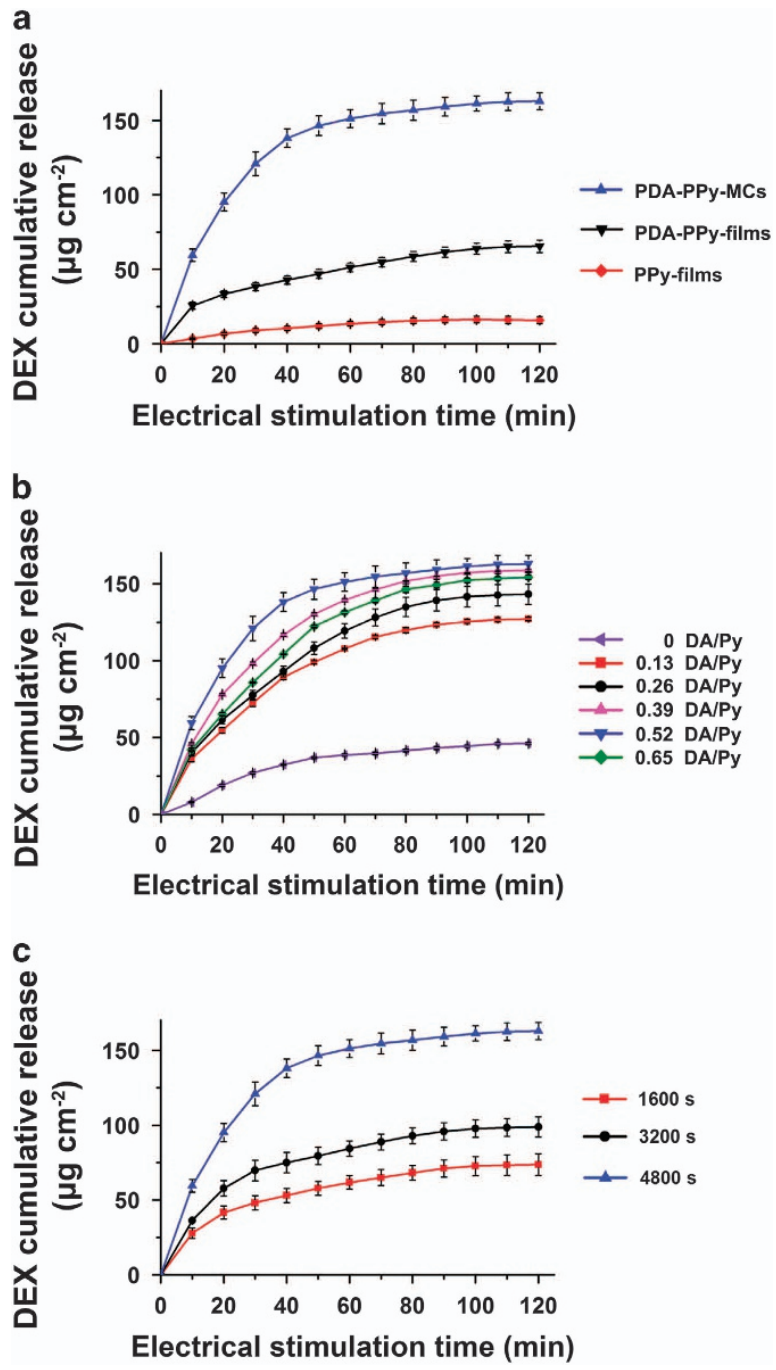

Figure 4 DEX cumulative release profiles in response to ES of (a) the PPy-films, PDA-PPy-films and PDA-PPy-MCs, (b) PDA-PPy-MCs with different DA/Py molar ratios and (c) PDA-PPy-MCs with different polymerization time.

On-demand drug delivery from PDA-PPy-MCs

Drug delivery from the electroresponsive and conductive PDA composite MCs could be flexibly controlled through on-off pulses of ES (Figure 5a). When ES was applied, a linear release profile of DEX was produced. When ES was off, almost no DEX was released from the MCs. The amounts of DEX released from the PDA-PPy-MCs can be tuned by altering the reductive potentials (Figure $5 \mathrm{~b}$ ). The amount of DEX released under $-1 \mathrm{~V}$ was $59.5 \pm 4.2 \mu \mathrm{g} \mathrm{cm}^{-2}$, which is twofold of that under $-0.5 \mathrm{~V}\left(24.6 \pm 1.4 \mu \mathrm{g} \mathrm{cm}^{-2}\right)$, and release reached to a maximum of $90.7 \pm 1.4 \mu \mathrm{g} \mathrm{cm}^{-2}$ when the potential is $-1.5 \mathrm{~V}$. The reason that higher DEX release was obtained at the higher potential of -1.5 is the majority of PPy backbone being in the reduced state at the higher potential. ${ }^{31}$ Therefore, more DEX were released at higher potential. Ideally, the higher potential was applied, the more DEX was released. However, the current was too large to keep the electrolyte stable when the negative potential was over $-1.5 \mathrm{~V}$. Cui's group also reported that the high potentials $(-2 \mathrm{~V})$ could impair the drug-release process because of the bubbles that were produced by the electrolysis of water. ${ }^{6}$ 

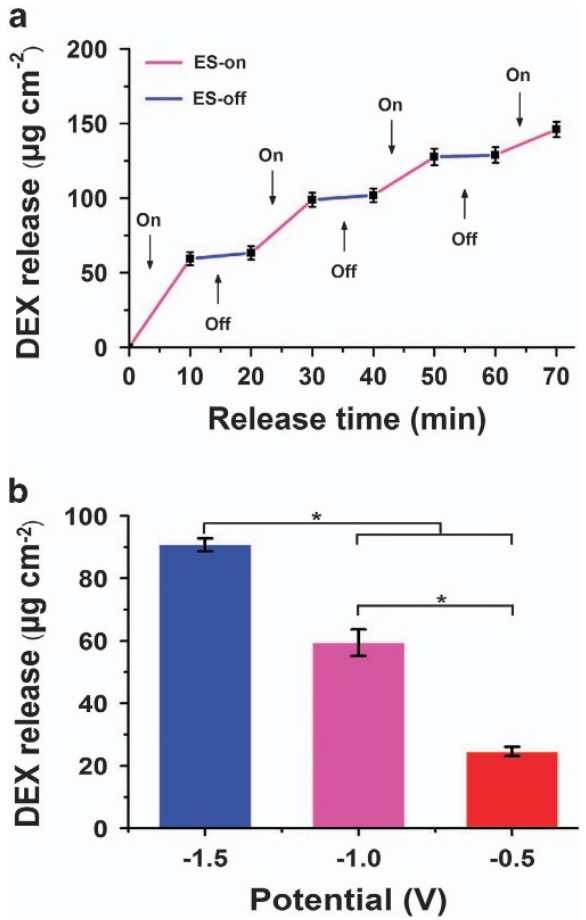

Figure 5 (a) On-demand delivery profile of DEX from PDA-PPy-MCs under -1 V. (b) Amount of DEX released from the PDA-PPy-MCs under different potentials for $10 \mathrm{~min}$.

High-throughput stimulation of BMSCs on PDA-PPy-MCs

The high-throughput stimulation study evaluated the synergistic effects of the PDA-PPy-MC surface composition, surface structures and ES potentials on BMSC activity (Figure 6a). The results indicated that PDA-PPy-MCs have a high cell affinity. Fluorescence staining images showed that BMSCs on PDA-PPy-films spread better than those on the pristine PPy-films and Ti (Figure 6b). A CCK-8 assay revealed that BMSCs on the PDA-PPy-films have a higher proliferation activity than those on the pristine PPy-films (Figure 6c). An ALP assay also showed that BMSCs have a higher differentiation activity on the PDA-PPy-films (Figure 6d).

Moreover, the microporous structure of the MCs favored cell adhesion and proliferation. BMSCs also spread well on the PDA-PPy-MCs (Figure 6b), and exhibited higher proliferation and differentiation than those on other flat surfaces (Figures $6 \mathrm{c}$ and $\mathrm{d}$ ). These results demonstrated that combining PDA and MC is effective for enhancing cell activity.

PDA-PPy-MCs can act as a platform for modulating cell behaviors through ES because of their good conductivity and cell affinity. BMSCs grew denser with an increase in the magnitude of the ES potentials from 0 to $900 \mathrm{mV}$ (Figure 6b). In addition, a CCK-8 assay also showed that the proliferation of the BMSCs increased with the increase in the magnitude of the ES potentials (Figure 6c). However, an ALP activity assay showed that the differentiation of the BMSCs reached its highest point under $300 \mathrm{mV}$. At higher ES potentials, the ALP activity dropped, indicating that a higher potential does not favor BMSC differentiation (Figure 6d). Combining these results, it is found that BMSCs have the highest proliferation and differentiation under optimal microstructural conditions, PDA content and ES signals.

\section{In vivo biocompatibility}

The PDA-PPy-MCs were prepared on porous $\mathrm{Ti}$ scaffolds to evaluate the biocompatibility of PDA-PPy-MCs in vivo. Histological analysis indicated that no inflammatory reactions were observable in all scaffolds after 4 weeks of subcutaneous implantation (Supplementary Information and Supplementary Figure S8). Fibrous tissue (FT) grew and integrated better in the PDA-containing porous Ti (PDA-PPy-films and PDA-PPy-MCs-coated porous Ti) scaffolds than on porous $\mathrm{Ti}$ scaffolds without PDA (pristine porous $\mathrm{Ti}$ and PPy film-coated Ti). PDA-PPy-MCs-coated porous Ti performed best among all groups, and the FT nearly filled the pores of the scaffold. These results demonstrated that PDA-PPy-MCs have good tissue affinity and biocompatibility. Moreover, the bioerosion of PDA-PPy-MCs were also evaluated in vivo (Supplementary Information and Supplementary Figure S9). After the 4 weeks of implantation, the tissue remained well integrated with the scaffold, and the PDA-PPy-MCs could be observed on the surface of the scaffold, revealing that the PDA-PPy-MC is biocompatible and stable in vivo.

\section{DISCUSSION}

In this study, PDA-PPy-MCs were produced to realize on-demand drug delivery using electrical therapy. Compared with traditional electroresponsive polymer-based composite materials, the PDA-PPyMCs have good biocompatibility, which can be ascribed to two aspects. One is the cell-affinitive PDA. PDA affords many functional groups to react with several biomolecules and proteins, particularly the signaling molecules on the cell membrane. The other is the microporous structure of the MCs. A microporous structure provides a similar extracellular matrix environment for the enhancement of biocompatibility to the biomaterial surfaces.

PDA, as a cell-affinitive molecule, was doped into PPy, thereby enhancing the cell affinity of the PDA-PPy-MCs. PDA affords many functional groups for reaction with a number of biomolecules and proteins, particularly the signaling molecules on the cell membrane, and is therefore widely used for the surface modification of biomaterials. Ku et al. ${ }^{32}$ prepared PDA coatings on four types of anti-adhesive substrates, including poly(dimethylsiloxane), silicone rubber, poly(tetrafluoroethylene) and poly(ethylene). They found that PDA promoted cell adhesion and viability on these substrates. Yang et al. ${ }^{33}$ used a biomimetic approach to coat PDA on various materials and found that PDA has effectively immobilized growth factor and promoted the proliferation and differentiation of human neural stem cells on PDA composite materials. Wu et al. ${ }^{34}$ used a self-assembly method to prepare a calcium phosphate/PDA composite nanolayer on the surface of a $\beta$-tricalcium phosphate scaffold. They also reported that the addition of PDA improved the attachment, proliferation and differentiation of human BMSCs. In the present study, BMSCs had higher proliferation and differentiation activity on the PDA-containing films (Figures $6 \mathrm{~b}$ and $\mathrm{c}$ ). These results are similar to the above studies, indicating that PDA in the PDA-PPy-films promotes their biocompatibility. The inclusion of PDA provides a new route to improve the cell affinity of conductive polymers and therefore may broaden the biomedical applications of a bundle of conducive polymers, such as polyaniline and poly(3,4-ethylenedioxythiophone).

The microporous structure of the PDA-PPy-MCs also favors cell attachment and growth. Polymer microcapsules possess functional groups and a high stability and are widely used for drug loading and cell encapsulation. ${ }^{35}$ Moreover, polymer microcapsules also have a microporous structure, which could modulate cell behaviors, such as adhesion, proliferation and differentiation. Liu et al. ${ }^{36}$ prepared 
a

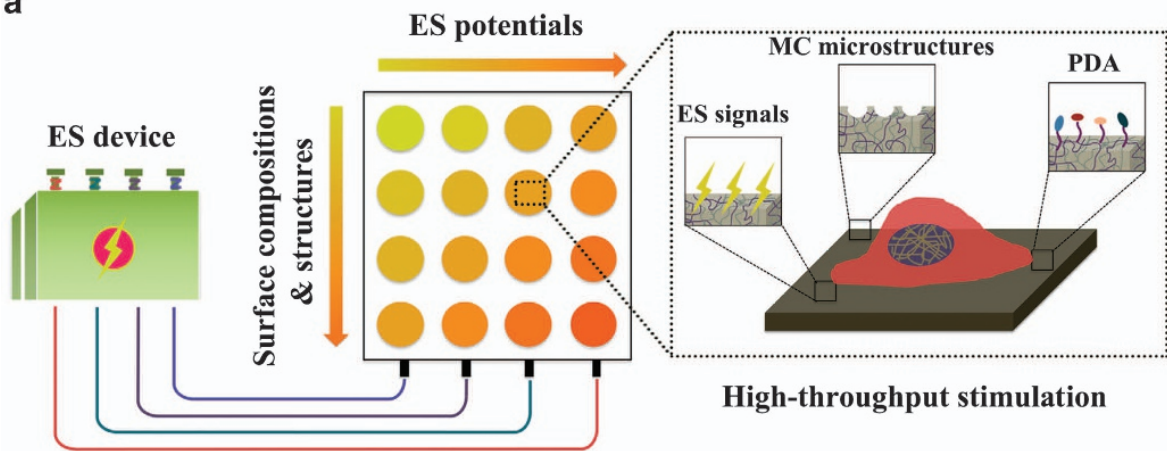

b
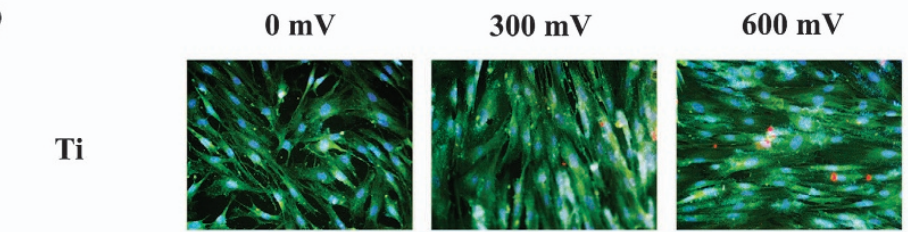

$900 \mathrm{mV}$

PPy-films
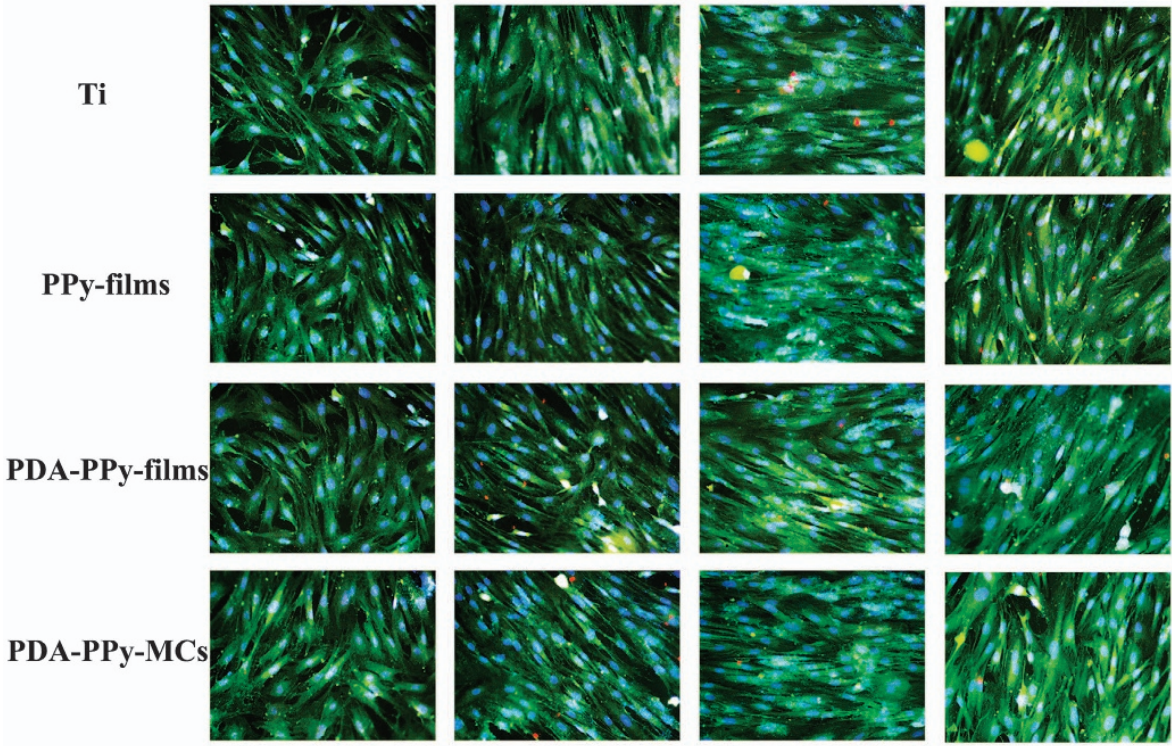

c
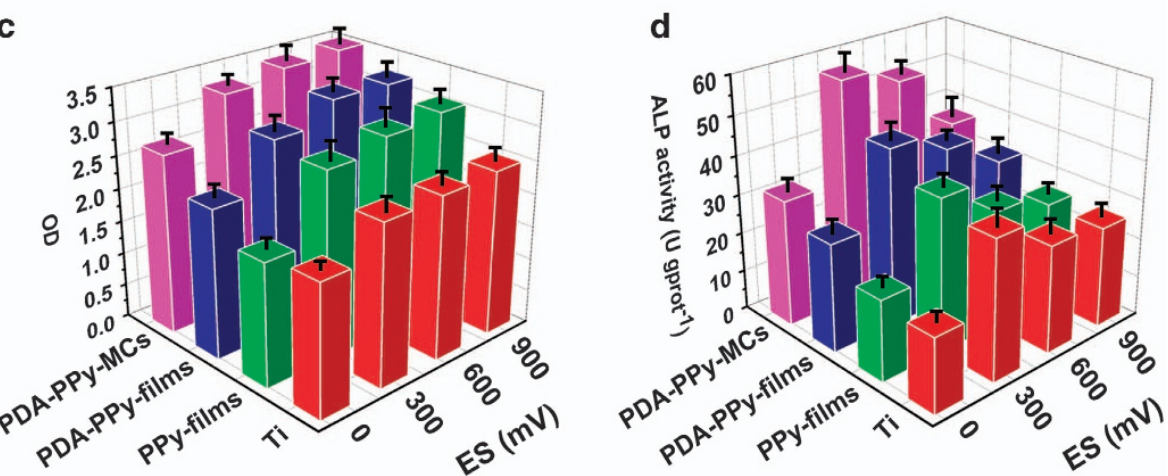

Figure 6 High-throughput stimulation of BMSCs on different surfaces with varying surface components, structures and ES potentials. (a) Schematics of highthroughput stimulation; (b) fluorescence staining images (7 days of culture), (c) proliferation (7 days of culture) and (d) differentiation (14 days of culture) of BMSCs on the surfaces of Ti, PPy-films, PDA-PPy-films and PDA-PPy-MCs under different ES potentials.

reduced graphene oxide/poly(methacrylic acid)-MCs on the surfaces of indium tin oxide and found that the microscale topography of the MCs promoted the adhesion of PC12 cells. In our previous study, we prepared PDA-MCs, which not only had a microporous structure but also enabled cell affinity. An in vitro study showed that the PDA-MCs enhanced the proliferation and osteogenic differentiation of BMSCs. ${ }^{23}$ In the present study, BMSCs have higher levels of cell activity in the PDA-PPy-MCs group than in other groups (Figure 6), revealing that a microporous structure is an important factor for improving cell adhesion. In summary, PDA-PPy-MCs have the advantages of both the cell-affinity properties of PDA and the microstructure of the MCs and can therefore effectively modulate cell behaviors through the synergistic effects of the PDA and MCs.

The ES effect of PDA-PPy-MCs was demonstrated by a highthroughput cell stimulation experiment that was carried on a homemade high-throughput stimulation system. The results of the high-throughput ES experiment showed that BMSCs exhibited high proliferation and ALP activities under ES, suggesting that 
PDA-PPy-MCs could promote the efficacy of electrical therapy. There are many reports on modulating cell behavior or repairing defective tissues through ES, such as cultured cells on CNT-containing scaffolds, ${ }^{37}$ Au nanoparticle-coated glass ${ }^{38}$ and PPy films ${ }^{39}$ under ES. However, there is no consensus on the optimal ES potential for cell proliferation and differentiation. This study found that the optimal ES potential for BMSC proliferation is $900 \mathrm{mV}$ and that for differentiation is $300 \mathrm{mV}$, suggesting that different levels of ES potential may modulate different cell activities and that there may be an optimal potential range for promoting cell growth and differentiation.

Furthermore, the high-throughput stimulation system evaluated the synergistic effects of the internal material factors (surface composition and surface structures) and the external physical factor (ES potentials) on BMSC activity. The results of the high-throughput ES study also showed that the microporous structure and PDA in the PDA-PPy-MCs strengthened the ES effect on cell activity. BMSCs demonstrated higher proliferation and differentiation on the microstructured surfaces (PDA-PPy-MCs) compared with non-microstructured surfaces (PDA-PPy-films) under the same ES conditions. In addition, BMSCs on the films contain PDA (PDA-PPy-films) have higher proliferation and differentiation than those on the films without PDA (PPy-films and Ti) under ES, which revealed that electrical signals combined with the cell-affinity properties of PDA could further modulate cell behavior. In summary, the electrical signals, surface microstructures and cell-affinitive molecules can synergistically improve cell growth.

The on-demand drug-delivery behavior of the PDA-PPy-MCs is dependent on the redox property of PPy. Compared with previous reports on PPy-based drug delivery, PDA-PPy-MCs have high drug-loading ability, which is ascribed to two factors. The first is the microporous structure of PDA-PPy-MCs that enhances the drug-loading capability. As shown in Figure 4, the maximum CRA of the microporous capsules (PDA-PPy-MCs) increased nearly 2.5-fold compared with dense PDA-PPy-films. The second is that PDA is used as the co-dopant with DEX to promote the available drug loading of PPy. ${ }^{40}$ When PDA was introduced into PPy-films, the maximum CRA increased from $15.8 \pm 2.3$ to $65.4 \pm 4.2 \mu \mathrm{g} \mathrm{cm}^{-2}$. There have been many reports on improving the drug-loading capability of PPy. However, they focus on either doping or producing a porous structure. For example, Weaver et al. ${ }^{9}$ used GO to load DEX by $\pi-\pi$ stacking and then co-doped the GO/DEX into PPy, thereby significantly improving the drug-loading capability of PPy. Luo et al. ${ }^{6}$ prepared PPy films with a nanoporous structure using a sacrificial template and found that the nanoporous films had a significantly higher drug-loading capability than non-nanoporous films. Our results suggest that combining PDA and a microporous structure is an effective method to enhance the drug-loading capability of PPy.

One interesting finding is that PDA incorporation improved the conductivity of PPy. Pristine PPy has a low conductivity, and doping is an effective method to improve its conductivity. The dopant, as a charge carrier, can remove or add electrons into the backbone of $\mathrm{PPy},{ }^{41}$ leading to a defect in the conjugated backbone of PPy. Thus, the electrons could be easily delocalized and freely move between the atoms. ${ }^{42}$ In this study, PDA was used as the dopant because PDA not only has a negative charge to neutralize the positive charge of the PPy backbone but also forms $\pi-\pi$ stacking between the PDA and PPy in favor of electron movement to further promote the conductivity of PPy (Scheme 1c). ${ }^{43}$ The conductivity of the PPy significantly increased from $3.2 \times 10^{-3}$ to $5.8 \times 10^{-2} \mathrm{~S} \mathrm{~cm}^{-1}$ when the PDA was doped into the PPy by the ED process. However, further increases in the DA/Py molar ratio lead to a decrease in the conductivity of the PPy because the excessive non-conducting PDA hinders the movement of electrons. For example, Zhang et al. ${ }^{44}$ doped DA into PPy via a chemical synthesis using protonic acid $(\mathrm{HCl})$ and an oxidant $\left(\mathrm{FeCl}_{3}\right)$. Their results also showed that the conductivity of PDA-doped PPy reaches a peak value of $42 \mathrm{~S} \mathrm{~cm}^{-1}$ and decreases to $0.05 \mathrm{~S} \mathrm{~cm}^{-1}$ with an increase in the DA/Py molar ratio from 0.032 to 0.64 . Our results corroborate this, further confirming that the incorporation of PDA enhances the conductivity of PDA-PPy-MCs while the DA/Py molar ratio is in an optimal range.

\section{CONCLUSIONS}

In this study, we developed electroresponsive and conductive PDA-PPy-MCs on the surfaces of electrodes that can locally and precisely release drugs and apply ES to modulate cell behaviors. Three features make the PDA-PPy-MCs beneficial for electrical therapy. First, PDA-PPy-MCs have a porous structure and good cell affinity to allow cell attachment and growth. Second, the electroresponsive PDA-PPy-MCs enable a high drug-loading capability of PPy. Third, PDA-PPy-MCs have good conductivity, which, together with their porous structure and cell affinity, means they can act as a conductive platform to apply electrical signals to stimulate cell behavior. Therefore, PDA-PPy-MCs have the potential to be applied for on-demand drug delivery and electrical therapy.

\section{CONFLICT OF INTEREST}

The authors declare no conflict of interest.

\section{ACKNOWLEDGEMENTS}

The work was financially supported by the 863 Program (2015AA034202), the National Key Research and Development Program of China (2016YFB0700802), NFSC (No. 81671824), Openfund of Key Lab of Advanced Technologies of Materials (MOE) and Fundamental Research Funds for the Central Universities (2682016CX075).

1 Famm, K., Litt, B., Tracey, K. J., Boyden, E. S. \& Slaoui, M. Drug discovery: a jumpstart for electroceuticals. Nature 496, 159-161 (2013).

2 Balint, R., Cassidy, N. J. \& Cartmell, S. H. Electrical stimulation: a novel tool for tissue engineering. Tissue Eng. Part B Rev. 19, 48-57 (2012).

3 Minev, I. R., Musienko, P., Hirsch, A., Barraud, Q., Wenger, N., Moraud, E. M., Gandar, J., Capogrosso, M., Milekovic, T. \& Asboth, L. Electronic dura mater for long-term multimodal neural interfaces. Science 347, 159-163 (2015).

4 Wenke, J. C. \& Guelcher, S. A. Dual delivery of an antibiotic and a growth factor addresses both the microbiological and biological challenges of contaminated bone fractures. Expert Opin. Drug Deliv. 8, 1555-1569 (2011).

5 Murdan, S. Electro-responsive drug delivery from hydrogels. J. Control. Release 92 , $1-17$ (2003)

6 Luo, X. L. \& Cui, X. T. Electrochemically controlled release based on nanoporous conducting polymers. Electrochem. Commun. 11, 402-404 (2009).

7 Luo, X. L. \& Cui, X. T. Sponge-like nanostructured conducting polymers for electrically controlled drug release. Electrochem. Commun. 11, 1956-1959 (2009).

8 Liu, Z., Robinson, J. T., Tabakman, S. M., Yang, K. \& Dai, H. Carbon materials for drug delivery and cancer therapy. Mater. Today 14, 316-323 (2011).

9 Weaver, C. L., LaRosa, J. M., Luo, X. L. \& Cui, X. T. Electrically controlled drug delivery from graphene oxide nanocomposite films. ACS Nano 8, 1834-1843 (2014).

10 Jayaprakash, N., Shen, J., Moganty, S. S., Corona, A. \& Archer, L. A. Porous hollow Carbon@ Sulfur composites for high-power lithium-sulfur batteries. Angew Chem. Int. Ed. Engl. 123, 6026-6030 (2011).

11 Santos, L., Martin, P., Ghilane, J., Lacaze, P. C. \& Lacroix, J. C. Micro/Nano-structured polypyrrole surfaces on oxidizable metals as smart electroswitchable coatings. ACS Appl. Mater. Interfaces 5, 10159-10164 (2013).

12 Sharma, M., Waterhouse, G. I., Loader, S. W., Garg, S. \& Svirskis, D. High surface area polypyrrole scaffolds for tunable drug delivery. Int. J. Pharm. 443, 163-168 (2013).

13 Aznar-Cervantes, S., Roca, M. I., Martinez, J. G., Meseguer-Olmo, L., Cenis, J. L., Moraleda, J. M. \& Otero, T. F. Fabrication of conductive electrospun silk fibroin 
scaffolds by coating with polypyrrole for biomedical applications. Bioelectrochemistry 85, 36-43 (2012).

14 Ateh, D., Navsaria, H. \& Vadgama, P. Polypyrrole-based conducting polymers and interactions with biological tissues. J. R. Soc. Interface 3, 741-752 (2006).

15 Meng, S., Zhang, Z. \& Rouabhia, M. Accelerated osteoblast mineralization on a conductive substrate by multiple electrical stimulation. J. Bone Miner. Metab. 29, 535-544 (2011).

16 Shi, Z. Q., Gao, H. C., Feng, J., Ding, B. B., Cao, X. D., Kuga, S., Wang, Y. J., Zhang, L. N. \& Cai, J. In situ synthesis of robust conductive cellulose/polypyrrole composite aerogels and their potential application in nerve regeneration. Angew. Chem. Int Ed. Engl. 53, 5380-5384 (2014).

17 Lee, H., Dellatore, S. M., Miller, W. M. \& Messersmith, P. B. Mussel-inspired surface chemistry for multifunctional coatings. Science 318, 426-430 (2007).

18 Rodriguez-Emmenegger, C., Preuss, C. M., Yameen, B., Pop-Georgievski, 0. Bachmann, M., Mueller, J. O., Bruns, M., Goldmann, A. S., Bastmeyer, M. \& Barner-Kowollik, C. Controlled cell adhesion on poly (dopamine) interfaces photopatterned with non-fouling brushes. Adv. Mater. 25, 6123-6127 (2013).

19 Cui, J., Yan, Y., Such, G. K., Liang, K., Ochs, C. J., Postma, A. \& Caruso, F. Immobilization and intracellular delivery of an anticancer drug using mussel-inspired polydopamine capsules. Biomacromolecules 13, 2225-2228 (2012).

20 Ochs, C. J., Hong, T., Such, G. K., Cui, J., Postma, A. \& Caruso, F. Dopamine-mediated continuous assembly of biodegradable capsules. Chem. Mater. 23, 3141-3143 (2011).

21 Chen, X., Yan, Y., Müllner, M., van Koeverden, M. P., Noi, K. F., Zhu, W. \& Caruso, F. Engineering fluorescent poly (dopamine) capsules. Langmuir 30, 2921-2925 (2014).

22 Shi, J., Yang, C., Zhang, S., Wang, X., Jiang, Z., Zhang, W., Song, X., Ai, Q. \& Tian, C. Polydopamine microcapsules with different wall structures prepared by a templatemediated method for enzyme immobilization. ACS Appl. Mater. Interfaces 5, 9991-9997 (2013).

23 Wang, Z., Li, C., Xu, J., Wang, K., Lu, X., Zhang, H., Qu, S., Zhen, G. \& Ren, F. Bioadhesive microporous architectures by self-assembling polydopamine microcapsules for biomedical applications. Chem. Mater. 27, 848-856 (2015).

24 Xie, C., Lu, X., Han, L., Xu, J., Wang, Z., Jiang, L., Wang, K., Zhang, H., Ren, F. \& Tang, Y. Biomimetic mineralized hierarchical graphene oxide/chitosan scaffolds with adsorbability for immobilization of nanoparticles for biomedical applications. ACS Appl. Mater. Interfaces 8, 1707-1717 (2016).

25 Yu, B., Liu, J., Liu, S. \& Zhou, F. Pdop layer exhibiting zwitterionicity: a simple electrochemical interface for governing ion permeability. Chem. Commun. 46, 5900-5902 (2010).

26 Cong, H. N., El Abbassi, K., Gautier, J. L. \& Chartier, P. Oxygen reduction on oxide/ polypyrrole composite electrodes: effect of doping anions. Electrochim. Acta 50, 1369-1376 (2005).

27 Zhang, W., Yang, F. K., Pan, Z., Zhang, J. \& Zhao, B. Bio-inspired dopamine functionalization of polypyrrole for improved adhesion and conductivity. Macromol. Rapid Commun. 35, 350-354 (2014).

28 Otero, T. F. Biomimetic conducting polymers: synthesis, materials, properties, functions, and devices. Polym. Rev. 53, 311-351 (2013).

29 Jiang, J., Zhu, L., Zhu, L., Zhu, B. \& Xu, Y. Surface characteristics of a self-polymerized dopamine coating deposited on hydrophobic polymer films. Langmuir 27, 14180-14187 (2011).

30 Xie, C., Lu, X. \& Wang, K. Pulse electrochemical synthesis of spherical hydroxyapatite and silver nanoparticles mediated by the polymerization of polypyrrole on metallic implants for biomedical applications. Part. Part. Syst. Charact. 32, 630-635 (2015).

31 Svirskis, D., Travas-Sejdic, J., Rodgers, A. \& Garg, S. Electrochemically controlled drug delivery based on intrinsically conducting polymers. J. Control. Release 146 6-15 (2010).
32 Ku, S. H., Ryu, J., Hong, S. K., Lee, H. \& Park, C. B. General functionalization route for cell adhesion on non-wetting surfaces. Biomaterials 31, 2535-2541 (2010).

33 Yang, K., Lee, J. S., Kim, J., Lee, Y. B., Shin, H., Um, S. H., Kim, J. B., Park, K. I., Lee, H. \& Cho, S. W. Polydopamine-mediated surface modification of scaffold materials for human neural stem cell engineering. Biomaterials 33, 6952-6964 (2012).

34 Wu, C., Han, P., Liu, X., Xu, M., Tian, T., Chang, J. \& Xiao, Y. Mussel-inspired bioceramics with self-assembled Ca-P/polydopamine composite nanolayer: preparation, formation mechanism, improved cellular bioactivity and osteogenic differentiation of bone marrow stromal cells. Acta Biomater. 10, 428-438 (2014).

35 Orive, G., Santos, E., Pedraz, J. \& Hernández, R. Application of cell encapsulation for controlled delivery of biological therapeutics. Adv. Drug Deliv. Rev. 67. 3-14 (2014).

36 Liu, H. W., Huang, W. C., Chiang, C. S., Hu, S. H., Liao, C. H., Chen, Y. Y. \& Chen, S. Y. Arrayed rGOSH/PMASH microcapsule platform integrating surface topography, chemical cues, and electrical stimulation for three-dimensional neuron-like cell growth and neurite sprouting. Adv. Funct. Mater. 24, 3715-3724 (2014).

37 Mooney, E., Mackle, J. N., Blond, D. J., O'Cearbhaill, E., Shaw, G., Blau, W. J., Barry, F. P., Barron, V. \& Murphy, J. M. The electrical stimulation of carbon nanotubes to provide a cardiomimetic cue to MSCs. Biomaterials 33, 6132-6139 (2012).

38 Park, J. S., Yang, H. N., Woo, D. G., Jeon, S. Y., Do, H. J., Huh, S. H., Kim, N. H., Kim, J. H. \& Park, K. H. Exogenous Nurr1 gene expression in electricallystimulated human MSCs and the induction of neurogenesis. Biomaterials $\mathbf{3 3}$, 7300-7308 (2012).

39 Hu, W. W., Hsu, Y. T., Cheng, Y. C., Li, C., Ruaan, R. C., Chien, C. C., Chung, C. A. \& Tsao, C. W. Electrical stimulation to promote osteogenesis using conductive polypyrrole films. Mater. Sci. Eng. C Mater. Biol. Appl. 37, 28-36 (2014).

$40 \mathrm{Liu}$, Y., Ai, K. \& Lu, L. Polydopamine and its derivative materials: synthesis and promising applications in energy, environmental, and biomedical fields. Chem. Rev. 114, 5057-5115 (2014)

41 Balint, R., Cassidy, N. J. \& Cartmell, S. H. Conductive polymers: towards a smart biomaterial for tissue engineering. Acta Biomater. 10, 2341-2353 (2014).

42 Ravichandran, R., Sundarrajan, S., Venugopal, J. R., Mukherjee, S. \& Ramakrishna, S. Applications of conducting polymers and their issues in biomedical engineering. J. $R$. Soc. Interface 7, S559-S579 (2010).

43 Xu, C. H., Sun, J. \& Gao, L. Synthesis of novel hierarchical graphene/polypyrrole nanosheet composites and their superior electrochemical performance. J. Mater. Chem. 21, 11253-11258 (2011).

44 Zhang, W., Zhou, Y. K., Feng, K., Trinidad, J., Yu, A. P. \& Zhao, B. X. Morphologically controlled bioinspired dopamine-polypyrrole nanostructures with tunable electrical properties. Adv. Electron. Mater. 1, 1500205 (2015).

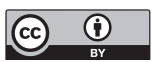

This work is licensed under a Creative Commons Attribution 4.0 International License. The images or other third party material in this article are included in the article's Creative Commons license, unless indicated otherwise in the credit line; if the material is not included under the Creative Commons license, users will need to obtain permission from the license holder to reproduce the material. To view a copy of this license, visit http:// creativecommons.org/licenses/by/4.0/

(C) The Author(s) 2017

Supplementary Information accompanies the paper on the NPG Asia Materials website (http://www.nature.com/am) 